\title{
EVALUACIÓN DEL EFECTO DEL IMPLANTE SYNOVEX-H SOBRE LA PREÑEZ DE NOVILLAS BRAHMAN CON UN MODELO DE REGRESIÓN LOGÍSTICA EXACTO
}

\section{EVALUATION OF THE EFFECT OF IMPLANT SYNOVEX-H ON BRAHMAN HEIFER PREGNANCY AN EXACT LOGISTIC REGRESSION MODEL}

\author{
Enrique Darghan $^{1} \mid$ Sinha Surendra ${ }^{2}$ Vitto Vega ${ }^{3}$
}

Forma de citar: DARGHAN Enrique, SURENDRA Sinha, VEGA Vitto. Evaluación del efecto del implante Synovex-H sobre la preñez de novillas brahman con un modelo de regresión logística exacto. Eco.Mat. 2014; 5(1): $16-26$.

Recibido:

Abril 15 de 2014

Aceptado:

Agosto 22 de 2014

\section{RESUMEN}

La presente investigación se realizó en la Hacienda Santa Rosa, centro genético de ganado Brahman del Táchira, Venezuela. En la investigación se analizó el efecto del Synovex-H sobre la preñez en primíparas de 24 meses de edad. Con el objeto de modelar la relación entre la preñez, el peso , año de monta y la aplicación del implante, se realizó un análisis de regresión logístico exacto utilizando como respuesta el diagnóstico de la preñez. De las variables explicativas, año, peso de monta e implante, resultaron con efecto significativo el peso de monta $(p<0,05)$ y el implante $(p<0,1$ en la estimación exacta y $p<0,05$ en la asintótica). Aunque el año del implante no resultó significativo, las novillas incorporadas el año 2004 tuvieron un porcentaje de preñez superior en $8,6 \%$ respecto al 2003. En lo que respecta al implante, se obtuvo porcentajes de preñez de $63,05 \%$ para implantadas y de $82,30 \%$ en las no implantadas. Este estudio soporta el concepto de que el peso de monta es benéfico sobre la reproducción de las novillas y a la vez muestra como el Synovex H tiene un efecto significativo desfavorable sobre la reproducción.

Palabras Clave: Regrasion logística exacta, Estimacion Máximoverosímil Condicional

\section{ABSTRACT}

The current investigation was made in the property Santa Rosa, genetic center of Brahman cattle in Táchira state, Venezuela. In the investigation the effect of the Synovex-H was analyzed on the pregnancy in primiparaes of 24 months of age. Of the explanatory variables, year, mounting weight and implant, resulted with significant effect, mounting weight $(p<0,05)$ and implant $(p<0,10$ in exact estimation and $p<0,05$ in the asymptotic). Although the year of implant was not significant, the heifers incorporated in the year 2004 had a pregnancy percentage superior in $8,6 \%$ with respect 
to 2003 . With regard to its implants, the pregnancy percentage of 63,05 was obtained for implanted and 82,30 for not implanted. This study supports the concept that the mounting weight is beneficial for the reproduction of the heifers and simultaneously it shows how the Synovex- $\mathrm{H}$ has an unfavorable significant effect on the reproduction.

Keywords: Exact logistic regression, Conditional Maximum- Likelihood Estimation

\section{INTRODUCIÓN}

$\mathrm{L}$ a eficiencia reproductiva de los bovinos es el factor de producción más importante en las explotaciones de ganado de carne, ya que de ella dependen directamente el ingreso económico y la calidad genética de los animales del rebaño. Por su gran importancia, la reproducción merece la mayor atención en las unidades de producción de ganado de carne, ya que su bajo valor a nivel tropical junto con la alta mortalidad y el atenuado ritmo de crecimiento, incide en los bajos niveles de productividad de la ganadería en general (Hoogesteijn et al., 1991). En hatos de cría, la eficiencia reproductiva tiene mayores efectos sobre la producción que el crecimiento de los becerros y la calidad de la canal. En bovinos tiene estrecha relación con la rentabilidad de la explotación. Las principales causas de pérdidas económicas se atribuyen principalmente al porcentaje de vacas vacías y a la mortalidad perinatal, por lo que resulta más rentable aumentar el número de becerros destetados que el peso mismo al destete, ya que éste es afectado principalmente por la edad y la raza (Basurto y Hernández, 2003).

La producción de carne en Venezuela depende de dos sistemas, el de doble propósito y el de cría para carne, ambos destinan animales al matadero o venden al sistema de ceba. Sin embargo, los índices productivos son diferentes entre si y superiores en el primero de los casos, ello debido a que el sistema doble propósito se ubica en zonas agro- ecológicas favorables, lo que facilita el establecimiento de prácticas de manejo pecuarias que permiten alcanzar mejores indicadores de producción. Por su parte, el de cría, de mayor impacto para la generación de carne en el país, se desarrolla fundamentalmente en condiciones de llano, donde las grandes extensiones de tierra, la estacionalidad climatológica, la diversidad del suelo y la calidad de los forrajes, dificultan la implementación de tecnológicas que conlleven a mejorar los indicadores de producción, en tal sentido, el ritmo de crecimiento es lento, lo que se traduce en bajo peso de ingreso a monta, provocando porcentajes de preñez poco productivos.

Estos problemas reproductivos, que se presentan en la ganadería de carne en Venezuela producto del bajo peso de ingreso a monta, inferior a $300 \mathrm{~kg}$, sugieren el uso de estrategias de fácil aplicación en el llano, que permitan incrementar la ganancia diaria de peso postdestete, llevando hembras a monta con peso superior a $300 \mathrm{~kg}$, mejorando la eficiencia reproductiva, lo que provocaría muy probablemente mayor productividad animal, más becerros generados para el sistema levante y ceba, y por ende, en el aumento de la carne producida. (Vitto, 2002).

Las investigaciones de la industria farmacéutica y la experiencia ganadera, han probado que ningún tipo de manejo del ganado proporciona más beneficios que las sustancias hormonales. Las mismas son empleadas por sus efectos anabolizantes, destinadas a
ISSN 1794-8231

PP: 16-26 
No. 1

Enero-Diciembre 2014 ISSN 1794-8231

PP: $16-26$ favorecer el crecimiento muscular e impactar en la conformación de la canal (Torrano, 2002). Ahora bien, es de esperar que los animales alcancen mayor ganancia de peso con las hormonas pues diversos trabajos así lo concluyen, pero su aplicación e impacto sobre vientres seleccionados para cría, tiene poca investigación, de hecho, en lo que respecta al uso de implantes hormonales y su efecto sobre la reproducción, se han reportado efectos secundarios por su utilización en hembras, entre ellos: prolapso vaginal, flojedad y edema vulvar, secreción excesiva de mucosa, estros prolongados sin prolapso vaginal y desarrollo de la glándula mamaria (Preston y Willis, 1975).

El Manual Merck de Veterinaria (1993) indicó que por haber retroalimentación negativa entre los niveles de estrógenos y los de LH(Hormona Luteinizante) y FSH(Hormona Folículo Estimulante), se puede afectar el inicio de la pubertad y la regularidad de los ciclos del celo, así como también reducir la tasa de fertilidad. Otras investigaciones presentan resultados mas restrictivos, tal es el caso en toros y novillas que se conserven o se vendan para ser destinados como reproductores, estos no deben ser implantados (Universidad de Purdue, 2002).

Todo lo antes descrito parece contradictorio cuando al comparar los índices productivos actuales con los que existían hace uno o dos decenios, los avances obtenidos resultan alentadores. En el campo de la alimentación animal destaca el auge del desarrollo y utilización de los promotores de crecimiento (Egaña, 2003).Por lo tanto, algunas investigaciones en la producción ganadera están avocadas al esclarecimiento respecto al uso apropiado de implantes no solo para lograr un mayor peso de los animales, sino también en base a sus resultados, proponer estrategias que fomenten el desarrollo de los diferentes sistemas productivos de carne, dentro de lo que se puede incluir sin temor alguno, el uso de promotores de crecimiento en novillas, justificando su uso por el interés en lograr mayor crecimiento que a su vez rinda más peso a la edad de monta y por ende mejor eficiencia reproductiva de las novillas.

Con base en estas razones, se realizó el presente experimento, utilizando el producto hormonal Synovex H (200 mg de propionato de testosterona y $20 \mathrm{mg}$ de benzoato de estradiol), de uso específico en hembras, con el fin de evaluar su efecto sobre la reproducción en vientres Brahman puros, de primera temporada de servicio y destinados a cría. La naturaleza de estas variables, específicamente la respuesta (diagnóstico de la preñez), sugiere el uso de un modelo de regresión logística, sin embargo, la falta de convergencia en la estimación máximo verosímil(intrínseca a este conjunto de datos) y el número de observaciones utilizadas, permite la aplicación de una propuesta de regresión logística apropiada, basada en la estimación máximo verosímil condicional, la cual es conocida como regresión logística exacta.

\section{MATERIALES Y METODOS}

El presente estudio se realizó en la Unidad Académica Hacienda Santa Rosa propiedad de la Universidad Nacional Experimental del Táchira, la cual está localizada en las inmediaciones de la base aérea Mayor Buenaventura Vivas, en el poblado de Santo Domingo, municipio Monseñor Fernández Feo, zona sur del estado Táchira. Esta unidad cuenta con 288 hectáreas destinadas a la explotación de ganado Brahman registrado.

En lo que respecta al rebaño bajo experimentación, se utilizaron hembras de la raza Brahman pura, debidamente registrados en la Asociación Venezolana de Criadores de Ganado Cebú (ASOCEBU) destinados para la producción de vientres para la generación de las crías F1 (Holstein $\times$ Brahman).

Por la superficie y los forrajes existentes, se estableció un programa alimenticio, basado en el pastoreo con rotación de potreros y suministro de minerales ad-libitum. Además, los animales post-destete recibieron alimento concentrado comercial, no individualizado, 
a razón de $1 \mathrm{~kg} /$ animal/día desde el destete hasta su venta como reproductores o ingreso a servicio, lo que ocurre a los 24 meses de edad. El programa reproductivo se lleva bajo la modalidad de temporada de servicios y el programa de inseminación artificial se utiliza como estrategia de mejoramiento genético, aplicándolo generalmente sobre vacas secas, de primer servicio y paridas temprano. Además, se realizan pruebas de fertilidad de los toros y exploración ginecológica vía rectal de las hembras, para evaluar estado reproductivo previo a la temporada de servicio y diagnóstico de preñez entre los 45 y 60 días de concluida.

La unidad de producción está ubicada en zona de vida de bosque húmedo tropical, a $330 \mathrm{msnm}$, con precipitación anual promedio de $2553 \mathrm{~mm}$ y temperatura media anual de $23,3{ }^{\circ} \mathrm{C}$, con poca diferencia entre meses. La humedad relativa es superior al $70 \%$ durante todo el año, con promedio anual de $81 \%$ (FAV, 2000).

Los suelos en su mayoría corresponden al orden de los entisoles, de formación aluvial, muy jóvenes de textura franco a franco arenosa, con un $\mathrm{pH}$ entre 5,3 - 6,1; con profundidad promedio de $20 \mathrm{~cm}$ y afloración de granzón en muchas áreas, con regular contenido de materia orgánica, buen drenaje superficial e interno y pobre fertilidad natural, características que limitan su uso con fines agrícolas y le dan su vocación pecuaria. La topografía es predominantemente plana, con ligeras ondulaciones y zonas quebradas, producto de la acción erosiva del río Uribante. El área dedicada a la recría, está delimitada por cuatro cursos permanentes de agua que sirven de drenaje natural y fuente de suministro de agua para los animales.

El principio activo del implante consiste en $200 \mathrm{mg}$ de propionato de testosterona y 20 $\mathrm{mg}$ de benzoato de estradiol. Según Neumann (1989), estas son hormonas naturales químicamente idénticas a las producidas en las glándulas endocrinas animales, las cuales se absorben, metabolizan y eliminan de la misma forma. La presentación comercial viene dada por dosis en comprimidos, con aproximadamente $3 \mathrm{~cm}$ de largo, de forma cilíndrica, constituidos por un núcleo inerte de goma de silicón, en el cual se incorporan los cristales de hormona para ser administrados por vía auricular subcutánea (Fernández et al., 2000). Se presenta en cartuchos de 10 dosis con 8 comprimidos cada una, durando su efecto unos noventa días (Gagrovet, 2002). Fort Dodge (2004) establece un periodo de 90 a 120 días de duración del efecto. La colocación del implante se realizó siguiendo la técnica recomendada por Neumann (1989).

En relación al efecto del Synovex $\mathrm{H}$ sobre la preñez, se evaluaron las mautas una vez que alcanzaron los 24 meses de edad y fueron incorporadas a su primera temporada de servicio. Para ello, se llevaron a monta 38 en abril del 2003 y 43 en abril del 2004. En total en los dos años que se evaluó la reproducción, a través de la preñez, se analizaron 81 novillas, de las cuales solo 41 fueron implantadas.

El peso de monta de las novillas fue considerado como covariable. Además, se tomó en cuenta el efecto año sobre la preñez, pues es bien conocido que este suele afectar no solo al porcentaje de preñez, sino también a otros caracteres reproductivos, pues es un reflejo de las diferencias establecidas entre años en relación al nivel nutricional, influenciado por el clima. En este caso fueron considerados los años 2003 y 2004. En total, se evaluó la presencia o ausencia de preñez en un total de 81 novillas de prácticamente la misma edad y del mismo grupo racial. En este sentido, es pertinente indicar que los animales de este estudio no fueron incluidos en manejo especial alguno, ellos permanecieron, como es costumbre en un solo lote y bajo la dirección normal de los programas llevados por el personal encargado en la unidad de producción.

Para llevar a cabo el análisis estadístico descriptivo, fue construida inicialmente una tabla cruzada con las frecuencias observadas de las novillas según resultaron o no preñadas y según la aplicación o no del implante Synovex H, con sus pesos de monta promedio
Enero-Diciembre 2014 ISSN $1794-8231$ PP: $16-26$ 
No. 1

Enero-Diciembre 2014 ISSN 1794-8231

PP: 16-26

según la combinación de niveles de los factores implante y año.

En lo que respecta al componente inferencial, fueron evaluados los efectos asociados al peso inicial al momento del ensayo, implante hormonal con Synovex H y año mediante un modelo de regresión logística exacto debido a la naturaleza binaria de la variable respuesta diagnóstico de la preñez y fundamentalmente debido a la falta de convergencia en la estimación máximo verosímil cuando surgen los reconocidos problemas de separación completa y cuasi-separación en los datos (ver King y Ryan, 2002). En el modelo de regresión logística exacto se considera como variable respuesta el diagnóstico de la preñez, y como variables explicativas a los factores implante (presente y ausente) y año (2003 y 2004) y a la variable Peso (al inicio del ensayo).

Para el análisis se utilizó un software especializado disponible en demo en Internet, el cual utiliza un algoritmo publicado por Mehta et al (1993) y que fue implementado en el paquete LogXact. Este algoritmo rinde los estimadores máximo verosímiles y máximo verosímiles condicionales de los parámetros del modelo así como las respectivas pruebas de bondad de ajuste, intervalos de confianza de los parámetros estimados y pruebas de hipótesis simples y conjuntas de los parámetros de interés. Estas pruebas producen un valor de significación $(p)$ exacto y uno medio, como consecuencia de la naturaleza discreta de la distribución. El modelo inicial involucró al intercepto así interacciones, y una vez observado el valor de $p$, se eliminaron del modelo todas aquellas interacciones que no resultaron significativas, de hecho, el modelo final solo involucró los efectos principales.

\section{RESULTADOS}

La tabla 1 muestraparatodas las combinaciones de las variables explicativas involucradas en el análisis, algunas estadísticas descriptivas

por celda, incluyendo al peso inicial(peso de monta) para los diferentes niveles de la respuesta (preñada o vacía). El par de valores en cada celda representan a la media (valor superior) y la desviación estándar del peso inicial para los grupos conformados. Además, de esta tabla puede calcularse fácilmente que del total de implantadas del año 2003(19 novillas), resultaron preñadas el $57,9 \%$ de las novillas (11 novillas), mientras que para el año 2004 resultaron preñadas el 68,2\%. Es importante destacar que en cada uno de estos años fueron implantados porcentajes prácticamente iguales de novillas, de hecho, en el 2003 fueron implantados el 50\% de las novillas disponibles para este año, mientras que para el año 2004 fue implantado el $51,2 \%$ de las novillas que fueron utilizadas para el ensayo. Respecto al total de novillas utilizadas en el experimento durante el par de años, puede observarse que un $72,8 \%$ se diagnosticaron como preñadas.

Tabla 1: Estadísticas descriptivas del peso de monta según año, implante y diagnóstico de preñez.

\begin{tabular}{|l|l|l|l|l|}
\hline & \multicolumn{2}{|l|}{ Implantada } & \multicolumn{2}{l|}{ No implantada } \\
\hline Año & preñada & vacía & Preñada & vacía \\
\hline 2003: media & 313,18 & 303,25 & 301,47 & 265,00 \\
\hline $\begin{array}{l}\text { 2003: desviación } \\
\text { estándar }\end{array}$ & 31,72 & 41,85 & 30,23 & 24,15 \\
\hline 2004: : media & 355,67 & 340,00 & 348,61 & 341,67 \\
\hline $\begin{array}{l}\text { 2004: desviación } \\
\text { estándar }\end{array}$ & 30,99 & 17,56 & 33,25 & 46,46 \\
\hline
\end{tabular}

Las tablas 2 y 3 describen la idoneidad del modelo. En primer lugar, la tabla 2, muestra los estadísticos de la bondad del ajuste, la deviancia y la razón de la verosimilitud junto con sus respectivos valores de significación. Si se denota con $\mathrm{D}$ al valor de la deviancia, se tiene que $\mathrm{D}=59,2645$ y su $p$-valor es 0,2578 , con lo cual se concluye que el modelo ajusta los datos adecuadamente (ausencia de sobre-dispersión).

En el caso de la razón de la verosimilitud, denotada con $\mathrm{QL}$, su valor es $\mathrm{QL}=26,5202$ 
y su valor de $p$ es $0,0000(p<0,01)$, con lo cual se rechaza la hipótesis nula de que todos los parámetros del modelo, incluyendo el intercepto, son simultáneamente cero. Con este último resultado se está probando la significación completa del modelo utilizado $\mathrm{y}$ en este caso se entiende que el ajuste del modelo es altamente significativo.

Tabla 2: Estadísticos de prueba para la bondad de ajuste del modelo de regresión logística exacta.

\begin{tabular}{|l|l|l|l|}
\hline Estadístico & Valor & g.l. & $\boldsymbol{p}$-valor \\
\hline Deviancia & 59,2645 & 53 & 0,2578 \\
\hline $\begin{array}{l}\text { Razón de la } \\
\text { verosimilitud }\end{array}$ & 26,5202 & 4 & 0,0000 \\
\hline
\end{tabular}

La tabla 3 muestra los estadísticos asintóticos de la razón de verosimilitud, Wald, Score y el estadístico exacto con sus respectivos $p$-valores. En esta tabla se observa que no fue significativo el efecto nulo conjunto de las variables explicativas controladas implante y año. La naturaleza discreta de la distribución utilizada sugiere el uso del $p$-medio en lugar del $p$-valor, sin embargo, ambos permiten llegar al mismo resultado en cuanto a que el efecto del implante y año en forma conjunta no son significativos. El código NA significa no disponible, pues en el caso de las pruebas asintóticas no está disponible el $p$-medio, al igual que los grados de libertad (g.l.) no lo están para la prueba exacta.
Tabla 3: Prueba de hipótesis conjunta para el efecto nulo de las variables explicativas implante y año.

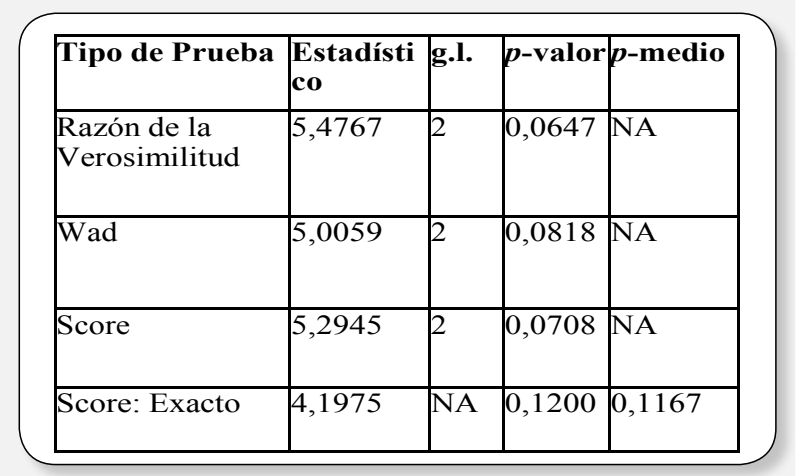

La tabla 4 muestra los parámetros estimados $(\beta)$ y sus errores estándar (e.e.) mediante el uso del análisis de regresión logística exacto, además, aparece el intervalo de confianza (IC) para el parámetro estimado y el valor de $p$ asociado a cada parámetro estimado. Fueron utilizados los niveles más altos de cada factor como niveles de referencia, es decir, al utilizar los códigos 0 y 1 entre corchetes para identificar los animales implantados y no implantados respectivamente, el código 1 se utiliza como referencia y el 0 aparece en la tabla de parámetros estimados, esto también aplica al efecto año. En cada uno de los efectos aparece no solo la estimación máximo verosímil (MLE) para el caso asintótico(A), además se muestra la estimación máximo verosímil condicional (CMLE) para el caso exacto.

Tabla 4: Tabla de parámetros estimados y sus respectivos intervalos de confianza y p-valores para el peso de monta (PM), implante (nivel: ausente (Imp[0])), año (nivel 2003: (Año[0])) y el intercepto.

\begin{tabular}{|c|c|c|c|c|c|c|c|}
\hline & \multicolumn{3}{|c|}{ Estimación puntual } & \multirow[t]{2}{*}{ Tipo } & \multicolumn{2}{|c|}{ Intervalo al } & \multirow{2}{*}{\begin{tabular}{c|}
$\boldsymbol{p}$-valor \\
bilatera
\end{tabular}} \\
\hline Término en el modelo & Tipo & B & e.e. & & inferior & superior & \\
\hline \multirow{2}{*}{ Peso de monta(PM) } & MLE & 0,0179 & 0,009 & A & 0,0004 & 0,0353 & 0,0449 \\
\hline & CMLE & 0,0172 & 0,009 & $\mathrm{E}$ & 0,0005 & 0,0351 & 0,0432 \\
\hline \multirow{2}{*}{$\begin{array}{l}\text { No implantada: } \\
\text { (Imp[0]) }\end{array}$} & MLE & 1,2560 & 0,568 & A & 0,1437 & 2,3683 & 0,0269 \\
\hline & CMLE & 1,0959 & 0,558 & $\mathrm{E}$ & $-0,0957$ & 2,4030 & 0,0760 \\
\hline \multirow{2}{*}{ Año: 2003 (Año[0]) } & MLE & 0,3506 & 0,659 & A & $-0,9407$ & 1,6419 & 0,5946 \\
\hline & CMLE & 0,3447 & 0,652 & $\mathrm{E}$ & $-1,1061$ & 1,8644 & 0,8381 \\
\hline \multirow[t]{2}{*}{ Intercepto } & MLE & $-5,4954$ & 3,129 & $\mathrm{~A}$ & $-11,6283$ & 0,6375 & 0,0790 \\
\hline & CMLE & $-5,4312$ & 8,207 & $\mathrm{E}$ & $-13,9829$ & 3,1145 & 0,7348 \\
\hline
\end{tabular}


No. 1

Enero-Diciembre 2014 ISSN 1794-8231

PP: $16-26$

\section{2}

Al utilizar las filas correspondientes a la estimación máximo verosímil condicional (CMLE), es claro que la covariable peso de monta (PM) resultó significativa tanto en la estimación exacta como en la asintótica.

En lo que refiere al implante, se obtuvo un efecto significativo $(p<0,10)$, con lo cual, implantar no tiene el mismo efecto sobre la preñez de los animales que el no implantar. El efecto año fue la única de las variables explicativas que no resultó significativa sobre la preñez $(p>0,05)$. La razón para usar el nivel se significación del $10 \%$ en el caso del implante se basa en este caso en la publicación del Doctor Thisted del Departamento de Estadística y Estudios de Salud de la Universidad de Chicago, quien afirma que según la naturaleza del estudio, el investigador puede establecer su nivel de significación. Además, en vista de que el valor es tan próximo al $5 \%$, es importante también observar el valor de la propuesta asintótica, en la cual el nivel de significación evaluado resultó inferior al 5\%.

Para evitar esta ambigüedad, la elección de un nivel que contenga las dos propuestas a la vez, sería la opción más conveniente en este caso, pues la diferencia en el porcentaje de preñez entre implantadas y no implantadas está alrededor del $20 \%$. Cuando el valor de $p$ es levemente superior al 5\%, algunos autores prefieren decir que el efecto es cercanamente significativo antes que concluir que no resultó significativo, obviamente, esto depende de la naturaleza de la investigación y de algunos otros resultados de la misma (Stokes et al., 2000).

Volviendo nuevamente al modelo, la expresión definitiva para el modelo de regresión logística exacto puede escribirse como:

$\log \mathrm{it}(\theta \mathrm{j})=1 \mathrm{og}(\theta \mathrm{j} /(1-\theta \mathrm{j}))=$ $5.4312+0.0172 \mathrm{PMj}+1.0959 \mathrm{Imp}[0]$ $\mathrm{j}+0.3447$ Año[0]j

donde, la cantidad $\theta \mathrm{j}$ es la probabilidad de que una novilla quede preñada en el $j$-ésimo grupo $(j=1,2, \ldots, 64)$ correspondiente con los niveles de los dos factores y los diferentes valores de la covariable peso de monta( los 64 grupos se originaron de los 81 datos, pues debe tenerse en cuenta que aunque solo hay dos niveles para implante y dos niveles para año, la mayoría de los pesos al inicio de la monta son distintos, con lo cual se reducen las 81 filas de datos a 64 filas donde la tripleta implante, año y peso son diferentes). Las variables Imp[0]j y Año[0]j toman el valor de uno (1) si el animal no fue implantado y si pertenece al grupo tratado en el año 2003, respectivamente, en caso contrario toman su valor en cero $(0)$.

En la tabla 5 se presentan los valores observados de la proporción de novillas que resultaron preñadas y las probabilidades pronosticadas ( $\hat{\theta}$ ) por el modelo según la característica de interés en la variable respuesta, que en este caso es un diagnóstico positivo de la preñez. En la primera fila se presenta la probabilidad observada de novillas preñadas para el peso de monta promedio correspondiente a la celda de novillas preñadas, y en la segunda fila aparece la probabilidad pronosticada con el modelo de regresión logística exacta.

A modo de ilustración, en la celda correspondiente a las novillas implantadas del año 2003, de la tabla 1 se obtuvo que el $57,9 \%$ de las novillas resultaron preñadas (11/ $(11+8))^{*} 100$ y del modelo se obtiene que con un peso promedio $\mathrm{PM}=313,18 \mathrm{~kg}$, en novillas implantadas, $\operatorname{Imp}[0]=0$, pertenecientes al año 2003, es decir, Año[0]=1, se tiene que:

$\log (\hat{\theta} /(1-\hat{\theta}))=$

$-5,4312+0,0172 * 313,18+1,095$

$9 * 0+0,3447 * 1=-0,3002$,

con lo que $\hat{\theta}=0,574$.

Es cierto que el peso sustituido a manera de ilustración no se corresponde con ninguno de los pesos de los 64 grupos conformados, sin embargo, el valor está dentro del rango de pesos que permitió el ajuste del modelo, además, puede notarse una muy aceptable proximidad entre el valor observado y el ajustado por el modelo para este caso en particular y para el resto de las celdas de la tabla 1, con lo cual se corrobora aún más el ajuste del modelo propuesto. 
Tabla 5: Valores observados y pronósticos de la probabilidad de preñez para el peso promedio de monta correspondiente solo al grupo de novillas que resultó preñada.

\begin{tabular}{|c|c|c|c|}
\hline \multicolumn{2}{|c|}{ Implantada } & \multicolumn{2}{c|}{ No implantada } \\
\hline $\begin{array}{l}\text { Año } \\
2003\end{array}$ & $\begin{array}{l}\text { Año } \\
2004\end{array}$ & $\begin{array}{l}\text { Año } \\
2003\end{array}$ & $\begin{array}{l}\text { Año } \\
2004\end{array}$ \\
\hline 0,579 & 0,682 & 0,789 & 0,857 \\
\hline 0,574 & 0,665 & 0,768 & 0,840 \\
\hline
\end{tabular}

\section{DISCUSIÓN}

El análisis correspondiente para la preñez presentó un porcentaje observado de $72,68 \%$ para el par de años de estudio, este valor es inferior al determinado por Montoni et al. (1995) quienes observaron $77 \%$, entre 1985 y 1993, pero superior al 68,82 \% medido por Pacheco (2005), desde 1992 hasta 2003, ambos trabajos realizados en la unidad de producción donde se desarrolló la presente investigación.

De las variables explicativas año de implante, condición implantada o no implantada y el peso al inicio de monta o a los veinticuatro meses de edad sobre el diagnóstico de la preñez, tuvieron un efecto significativo el peso al inicio de la monta y el implante, mientras que el efecto año no resultó significativo. Aunque el año del implante no tuvo efecto significativo, las novillas incorporadas a la temporada de servicio en el año 2004 tuvieron un porcentaje de preñez superior en $8,6 \%$ respecto al año 2003 . El que el efecto año no resultara significativo, difiere de los resultados de Pacheco (2005), quien halló un efecto significativo debido a la temporada de servicio sobre el porcentaje de novillas preñadas, pues el autor observó valores que oscilaban según el año desde $31 \%$ al $100 \%$, lo cual pudiera atribuirse según el mismo autor, a las condiciones de manejo, disponibilidad de nutrientes y mejoras ambientales ofrecidas. Así mismo, en condiciones tropicales, el efecto del año de la temporada de servicio sobre la preñez ha sido estudiado por diversos autores (Cruz et al., 1971; Linares et al., 1974; Plasse et al. 1978; Romero, 1989; Montoni et al. 1992, Plasse et al., 1995;
Cárdenas, 2000; Vitto, 2002; Patiño, 2003) quienes hallaron resultados encontrados en cuanto al efecto significativo que puede tener el año de servicio sobre la reproducción de las novillas.

Como se señaló anteriormente, la aplicación del implante con Synovex-H presentó efecto significativo sobre la preñez $(p<0,10)$ o cercanamente significativo al $5 \%$ en la propuesta de regresión logística exacta, de hecho, los porcentajes de preñez fueron de $63,05 \%$ para hembras implantadas y de $82,50 \%$ en las no implantadas, observándose una diferencia porcentual de $19,45 \%$ en la preñez de los vientres no implantados sobre los implantados, en la muestra de 81 novillas. Estos resultados no son contradictorios con los de la tabla 3, donde el implante no resultó significativo, ya que la prueba no se está haciendo solo para el efecto implante sino para el efecto implante y año de manera conjunta, y como se discutió previamente, el efecto año no resultó significativo, pero ocurrió lo contrario para el efecto implante. Los porcentajes de preñez obtenidos son similares con los reportados por Henricks et al., citados por Araujo-Febres (1989), quienes hallaron fertilidad del $52 \%$ en novillas implantadas contra $80 \%$ del grupo control. Sin embargo, difieren de los de Kay et al. (1977), quienes lograron que todas las hembras implantadas a las 31 semanas de vida, se preñaran, a pesar de que presentaron retraso en la aparición de la pubertad.

De la misma manera, los valores de preñez encontrados en la actual investigación son inferiores a los encontrados por Morrison, citadoporNorden(1987), pues las implantadas, después de los seis meses extra de crecimiento antes de la monta, tuvieron tasa de concepción del $85 \%$. Por otro parte, se podría indicar que son mejores que los resultados encontrados por Hoffman et al. (1988) ya que en este caso, las novillas implantadas no presentaron estro. En este sentido, y considerando la propuesta de regresión logística exacta, la cual es conveniente en muestras pequeñas( como nuestro caso) y utilizando un nivel de significación del $10 \%$, se puede finalmente
Enero-Diciembre 2014 ISSN 1794-8231 PP: $16-26$ 
No. 1

Enero-Diciembre 2014 ISSN 1794-8231

PP: 16-26 concluir que el Synovex-H, tuvo un efecto significativo desfavorable sobre la preñez, lo cual puede atribuirse a que su aplicación suele afectar el desarrollo reproductivo de las hembras y la normalidad del ciclo sexual, limitando su capacidad reproductiva, lo cual es corroborado por Newman (1989) quien señaló que en las hembras, aunque es posible estimular el crecimiento, también se logra suprimir el estro; o por lo publicado por el Manual Merck de Veterinaria (1993), en relación a que por haber retroalimentación negativa entre los niveles de estrógenos y los de LH y FSH, se puede afectar el inicio de la pubertad y la regularidad de los ciclos del celo o reducir la tasa de fertilidad.

En todo caso, a la luz de los resultados y considerando que la presente investigación se desarrolló con la finalidad de medir el efecto que el implante tendría sobre la preñez, no queda sino indicar que la implantación de hembras destinadas a cría fue negativo para su reproducción, soportando lo señalado por Araujo-Febres (1989), de que no hay ninguna indicación que esta práctica manifieste alguna ventaja, o lo recomendado por Cajal y Romero (1988), en cuanto a que los implantes deben ser utilizados sólo en animales destinados al consumo y nunca en toros, vacas o novillas de reemplazo, ya que podrían afectar su comportamiento reproductivo.

Así también lo encontrado por la Universidad de Purdue (2002), en relación a que los toros y novillas que se conserven o se vendan para ser destinados a reproductores no deben ser implantados.
Como se discutió anteriormente, la variable explicativa peso de monta afectó significativamente la preñez $(p<0,05)$, coincidiendo con los resultados de Plasse et al. (1995), quienes observaron que para todas las edades el peso al inicio de la temporada de servicio influyó el porcentaje de preñez. Los resultados indican que mientras más pesados sean los animales al inicio de la temporada de servicio, mayor porcentaje de preñez alcanzarán. Estos resultados son similares con los de otros autores (Plasse et al., 1989; Montoni et al., 1992; Martínez et al., 1994; Pacheco, 2005) quienes evaluando el efecto del peso de los vientres al inicio de su primera temporada de servicio, hallaron relación entre el peso y la preñez, siendo mayor el porcentaje de gestación en novillas más pesadas.

Los resultados obtenidos en los valores observados, permiten evaluar el comportamiento que mostraron los vientres del ensayo en cuanto a preñez se refiere. Resalta el escaso porcentaje mostrado por las hembras implantadas, incorporadas a monta con menos de $300 \mathrm{~kg}$ de peso, es decir, los animales más afectados por el implante, desde el punto de vista reproductivo, fueron aquellos cuya ganancia de peso diaria resultó inferior a $370 \mathrm{~g}$, grupo de peso que indiscutiblemente, pudiera ser influyente en el impacto negativo global del efecto implante. Por otro lado, los animales con ganancias de peso superiores a los $440 \mathrm{~g}$ /día, que alcanzaron más de $350 \mathrm{~kg}$ de peso a los dos años de edad, se preñaron en porcentaje similar al grupo no implantado, por lo tanto, se debería tomar en cuenta el ritmo de crecimiento individual del animal, si se desean realizar otras pruebas de implantes hormonales de crecimiento sobre reproducción, evitando utilizar hembras de escaso desarrollo.

Los resultados obtenidos, en relación al impacto positivo del mayor peso de monta sobre la preñez, deberían estimular a productores, investigadores y entes gubernamentales a buscar estrategias de manejo de fácil aplicación de acuerdo a las condiciones de cría en Venezuela, que permitan aumentar el ritmo de crecimiento y por ende los índices reproductivos, lo que se traduciría en mayor cantidad de carne generada.

\section{REFERENCIAS}

Araujo, F. O. (1989). Experiencia con promotores de crecimiento (implantes) en Venezuela. V Cursillo sobre Bovinos de Carne. Universidad Central de Venezuela. UCV, Maracay, Venezuela. pp. 145-174. 
Basurto, H. y Hernández, I. (2003). Sincronización de estro en bovinos en Condiciones tropicales. Reproducción Animal, Centro de Enseñanza, Investigación y Extensión en Ganadería Tropical. Facultad de Medicina Veterinaria y Zootecnia. Universidad Nacional Autónoma de México.

Cajal, C. y Romero, H.(1988). ¿Qué son losimplantes?.http://www.patrocipes.uson. mx/invpec/RA0041,10/11/03.

Cárdenas, O (2000). Evaluación de la eficiencia reproductiva a través del porcentaje de pariciones en un rebaño Brahman ubicado en la zona de bosque húmedo tropical. Trabajo de Grado para optar al titulo de Ingeniero de Producción Animal. Universidad Nacional Experimental del Táchira. San Cristóbal Venezuela. pp. 10-35.

Cruz, V., Warnick, A. y Franke, D.(1971). Factores que afectan la reproducción de ganado de carne. ALPA.Mem. 6: 163.

Egaña, J. (2003). Anabolizantes en la carne. Escuela de Ciencias Veterinarias. Universidad de Chile. http://www.creces.cl//new/index. asp. 09/10/06.

FAV.(2000). Servicio de Meteorología, Departamento de Climatología. Estación de la Base Aérea Mayor Buenaventura Vivas Guerrero. Fuerzas Aéreas Venezolanas. Santo Domingo, Estado Táchira.

Fernández, C., Anastasio, D., Spassova, E. (2000). Avalicao e gestao de risco do uso de hormonas como promotores de crecimiento nas especies pecuarias. Facultad de Medicina Veterinaria. Universidade Técnica de Lisboa, seccao de morfología e clínica.

Hoffman, M., Strohbehn, D. y Mahdi, A.(1988). Implanting feedlot heifers with melengesterol acetate. J. Anim. Sci. 66: 452.

Hoogesteijn, R., Illesca, M. Mendoza, O. (1991). Programa de mejoramiento de la eficiencia reproductiva en dos hatos de cría extensiva en el Estado Apure. VII Cursillo sobre Bovinos de Carne. Universidad Central de Venezuela, Facultad de Ciencias Veterinarias. Maracay, Venezuela. pp. 113150.

Kay, R., Mallinson, C. and Little, W. (1977). Growth rate, feed conversion ratio and age of puberty of dairy heifers implanted with anabolic steroids. Anim. Prod. 24:133.

King, E. N. y Ryan, T. P. (2002). A Preliminary Investigation of Maximum Likelihood Logistic Regresion versus Exact Logistic Regresion. The American Statistician, Vol. 56, No. 3, pp. 163-170.

Linares, T., Plasse, D. Burguera, M. Ordoñez, J., Ríos, J., Verde, O. y González, M. (1974). Comportamiento productivo de Bos taurus y Bos indicus y sus cruces en el llano Venezolano. 1. Eficiencia reproductiva. ALPA Mem. 9: 289-301.

Manual Merck de Veterinaria.(1993). IV Edición, publicado por Merck \& Co INC. Grupo Editorial Oceano S.A., Barcelona, España. 1763 p.

Martínez, G., Lavandeira, L. Montes, L., Scull, J. y Benítez, R. (1994). Evaluación clínica reproductiva de la hembra Cebú en monta natural. 2. Efecto de los factores no genéticos en la tasa de gestación. Rev. Cub. Reprod. Anim. 20 (1): 35-46.

Mehta, C. R., Patel, N. R. and Jajoo, B. (1993). Exact logistic regression: theory, methods, and software. Cytel Software Corporation Technical Report. 1993.

Montoni, D., Rojas, G., Verde, O., Silva, J. y Arriojas, M. (1992). Producción de un rebaño Brahman bajo condiciones de trópico húmedo. I. Eficiencia reproductiva. Revista de la Facultad de Agronomía. UCV. Maracay, Venezuela. 18: 225-245.

Montoni, D., Vitto, J. y Rojas, G. (1995). Estrategia para el manejo de la vaca de primer parto en bovinos de carne. En: D.
ISS 1794-8231

PP: $16-26$ 
No. 1

Enero-Diciembre 2014 ISSN 1794-8231

PP: 16-26

Plasse., N. Peña de Borsotti y R. Romero. (Eds.).

XI Cursillo sobre Bovinos de Carne. Universidad Central de Venezuela, Facultad de Ciencias Veterinarias Maracay, Venezuela. pp. 161-195.

Neumann, A.(1989). Ganado vacuno para producción de carne. Editorial Limusa. I Edición. México. pp. 653-671. Norden, N. 1987. Novillas implantas destetan becerros más pesados.

Pacheco, I. (2005). Evaluación de algunos efectos genéticos y no genéticos sobre la preñez de vacas Brahman de primer servicio, en un rebaño ubicado en el Suroeste Andino. Trabajo de Grado. Universidad Nacional Experimental del Táchira. San Cristóbal, Venezuela. pp. 35-42.

Patiño, J. (2003). Evaluación de la tasa de preñez de vientres Brahman servidos a los dos años de edad en Hatos del Suroeste de Venezuela. Trabajo de Grado. Universidad Nacional Experimental del Táchira. San Cristóbal - Venezuela. pp. 55-60.

Plasse, D., Linares, T. Verde, O. y Bastidas, P.(1978). Factores que influyen la concepción en la primera lactancia en vacas Brahman. ALPA. Memoria 13: 167.

Plasse, D., Fossi, H. y Verde, O. (1989). Factores que influyen la preñez en bovinos de carne de primer servicio.V Cursillo sobre bovinos de Carne. Universidad Central de Venezuela, Facultad deCiencias Veterinarias.

Plasse, D., Verde, O.,Beltrán, J., Hernández, A., Márquez, N., Capriles, A., Arriojas, L., Shultz, T., Braschi, N. y Benavides, A. (1995). Tendencias anuales de producción e influencias genéticas y ambientales en un rebaño Brahman genéticamente cerrado. 4. Porcentaje de preñez, parición, destete, disponibilidad a 18 meses y producción por vaca. ALPA. 3(2): 113-130.

Preston T. y Willis, M. (1975). Producción intensiva de carne. Editorial Diana-México. pp. 378-395.

Romero, R. (1989). Estudio genético de caracteres reproductivos en vacas Brahman, Guzerá, Nelore y sus cruces. Tesis de Maestría. Universidad Central de Venezuela. Venezuela. pp. 75-90. 